\title{
Role of Cloud Computing in Creating a Sustainable Green ICT Infrastructure
}

\author{
Mohammed Khalid Kaleem \\ Department of Informatics, \\ Wollega University, Nekemte, \\ Ethiopia
}

\author{
Rituraj Jain \\ Department of Electrical and \\ Computer Engineering, Wollega \\ University, Nekemte, Ethiopia
}

\author{
Manaullah Abid Husain \\ Department of Electrical \\ Engineering, Jamia Millia \\ Ismalia, New Delhi, India
}

\begin{abstract}
The ICT sector has grown exponentially in the last two decades, transforming society and economy. The emission of carbon levels to the environment has also widely increased with the expansion of ICT sector. Cloud computing is a new model for providing business and IT services. The cost advantages of cloud computing have enabled new software vendors to create productivity application that they can make available to people at a much smaller cost than would be possible to shrinkwrapped software. This essay gives an overview of the importance of cloud computing in creating a sustainable green ICT infrastructure.
\end{abstract}

\section{Keywords}

Cloud Computing, Green ICT, ICT Sector, IT Services, Sustainability.

\section{INTRODUCTION}

Cloud computing has the potential to provide even relatively small companies with access to supercomputing power and highly advanced software tools, at relatively low cost through the Internet.

Cloud computing builds on so many older concepts in computer technology that it can be hard for people newly introduced to the concept to grasp that it represents a paradigm shift in computing it's an evolutionary change that enables a revolutionary new approach to how computing services are produced and consumed.

Cloud computing has shifted the economics of software delivery in a manner similar to the way that music downloads have shifted the delivery of commercial music. The cost advantages of cloud computing have enabled new software vendors to create productivity application that they can make available to people at a much smaller cost than would be possible to shrink-wrapped software [1]

\section{THE INFLUENCE OF CLOUD COMPUTING IN 3G ERA}

Ever since $3 \mathrm{G}$ services are launched by the major communication operators, the simple voice and information service can no longer meet the growing requirements of users. The 3G data services have become the focus of competition among operators. Many operators have introduced some specialized services. And with the growth of $3 \mathrm{G}$ clients and the expansion and improvement of $3 \mathrm{G}$ networks, operators have to

Cloud computing is a new model for providing business and IT services. The service delivery model is based on future development consideration while meeting current development requirements. The three levels of cloud computing Service (laaS, PaaS and SaaS) cover a huge range of services. Besides computing and the service delivery model of storage infrastructure, various models such as data, software application, programming model etc. can also be applicable to cloud computing More importantly the cloud computing model involves all aspects of enterprise transformation in its evolution, so technology architecture is only a part of it, and multi-aspect development such as organization. Processes and different business models should also be under consideration. Based on standard architecture methodology with best practices of cloud computing, a cloud Model Application Methodology can be used to guide industry customer analysis and solve potential problems and risks emerged during the evolution from current computing model to cloud computing model. This methodology can also be used to instruct the investment and decision making analysis of cloud computing model, determine the process, Standard, interface and public service of IT assets deployment and management to promote business development. The diagram below shows the overall status of this methodology provide more diversified $3 \mathrm{G}$ services to survive in the fierce market competition. Cloud can be used as a platform to provide such value added services [2].

In this $3 \mathrm{G}$ era, Mobile TV, mobile securities and data backup will all become critical businesses. Huge amounts of videos, images and documents are to be stored in data centers so that users can download and view them at any time, and they can promote interaction. Cloud computing can effectively support this kind of business requirements, and get maximal storage with limited resources. Besides, it can also search and provide the resources that are needed to users promptly to meet their needs [3].

After the restructuring of operators, the businesses of leading service providers will all cover fixed network and mobile service, and they may have to face up to fierce competition in $3 \mathrm{G}$ market. Cloud computing can support unify monitoring and dynamic deployment of resources. So, during the business consolidation of the operators, the cloud computing platform can deploy necessary resources in time to support business development, and respond quickly to market requirements to help operators to gain larger market share [4].

The 3G-enabled high bandwidth makes it easier and quicker to surf Internet through mobile phones and it has become a critical application of $3 \mathrm{G}$ technologies. Cloud computing makes it compatible among different equipment, software and networks, so that the customers can access the resources in the cloud through any kinds of clients [5].

\section{THE NEED FOR GREEN ICT}

Climate change is now the greatest challenge facing the global community, having serious implications on the future of world economy, health \& safety, food, security and many other dimensions. Rising levels of greenhouses gases such as carbon dioxide and methane have led to serious and irreversible changes in the earth's ecosystems. Il is generally accepted that 
increasing levels of greenhouse gases (GHG) in the earth's atmosphere are causing a continual rise in the earth's average surface temperature - by some estimates, average temperatures an increase by almost $5^{\circ} \mathrm{C}$ by the end of the $21^{\prime}$ 'st century as compared to the year 2000, Any rise in temperatures above 2'C can cause irreparable damage to Planet Earth and humanity. The ICT community is actively participating in the global scale efforts now underway in meeting this challenge. The ICT community has recognized the need for continuous improvements, new technologies and disruptive innovations in achieving the climate change related targets in front of the global community. Green ICT is the community's contribution towards this momentous task. Indeed. ICI is essential to make it economically possible to achieve necessary reduction in GHG concentration levels in the earth's atmosphere [6].

\section{CARBON FOOTPRINT OF ICT SECTOR}

The ICT sector has grown exponentially in the last two decade, transforming society and economy. ICT impacts business, lifestyle and family relationships like never before. As the ICT sector grows, the carbon footprint of the ICT sector will continue to grow. Here are some examples of the explosive growth of ICT:

- It is estimated that there will be more than 3 billion computers connected the Internet by 2011. There are predictions that by 2020 . The number of devices connected to the internet will be around 50 billion, forming part of the so called internet of things. Today there are more than 1.5 billion users of internet. This number is expected to increase significantly at more and more users from developing nations start using internet, many of whom will access Internet via their mobile phones.

- $\quad$ The number of mobile phone users in India in June 2009 was above 470 Million with an annual growth of almost $50 \%$

- For most economies, the share GDP attributable to the ICT sector is already quite significant and is increasing each year. In India, ICT sector contributed about $5.8 \%$ of the national GDP in FY2009. Share of GDP contributed to ICT sector or in developed economics such as UK is close to $7 \%$.

- As of 2007, The ICT sector was responsible for about $2 \%$ of total carbon emissions an over 0.8 billion tons of $\mathrm{CO} 2$ equivalent. With the kind of growth happening in the ICT sector. Total emissions from this sector are estimated to rise to about I. 4 billion tons by 2020 .

\section{MAJOR CONTRIBUTORS OF EMISSION FROM ICT SECTOR}

\subsection{Telecom infrastructure and Devices}

As of 2007, 37\% of all 3 emissions are due to telecom Q and devices. This includes emission caused by mobile network infrastructures, mobile devices, and fixed broadband and narrowband devices. Increasingly as more and more people get access to mobile telephony. The share of emissions from mobile devices and infrastructure will increase and will constitute almost $17 \%$ of all ICT emissions.

\subsection{Data Centers}

As of 2007, 14\% of all ICT emission is caused by data centers. This includes both corporate data centers and the Internet data centers where large scale consumer web applications such search engines and social networking sites are hosted roughly
$50 \%$ of the Emission due to data waters is due to power system losses and cooling loads. The hulk of the remaining part of the emission is caused by the energy consumed to power up low cost commodity servers that now dominate most data centers.

\subsection{End User Devices such as PCs and Peripherals}

Bulk of all ICT emissions is due to PCs. Desktops, laptops and peripherals such as printers. As of 2007, roughly $50 \%$ of all emissions are due to PCs and peripherals mainly because of the power consumed in CRT displays. By 2020, it is expected that there will be no CRT displays in use. However, as the number of PCs and laptops are expected to rise to around 4 billion. Total emissions from PCs and peripherals will increase in both absolute and percentage terms. By 2020, PCs and peripherals will contribute about $57 \%$ of all ICT emissions.

\subsection{Generic Approaches for Green ICT}

Having set the context of Green ICT, we now take a look at some generic approaches for reducing energy consumption ICT infrastructure.

\subsection{Increasing Visibility}

Insight into what is happening in the infrastructure at multiple levels of absorption and detail is a key requirement. One of the first things that must be put in place is $\mathrm{n}$ systematic approach towards energy consumption measurement and a set of performance metrics that capture energy usage efficiency Measurements and performance metrics are important because

- It enables energy use reduction via change in usage patterns by the consumer. When consumers are aware at a sufficient level of detail, they may adapt, reduce or altogether avoid energy consumption as appropriate.

- It clearly brings out inefficiencies in the system and encourages technology refreshes and optimal system design

- It enables automation in energy saving. Once a measurement system is in place energy optimization systems may be put in place that automatically acts the system operating points in a way that maximum useful work is obtained for the energy expended.

A good measurement system in a data center scenario would provide the following types of reports:

- Component wise consumption for each server, storage system and switch.

- Rack level power consumption.

- Subnet wise power consumption.

- Application wise power consumption.

- Customer/department wise power consumption.

In case of Telecom infrastructure, there is a need for making visible how energy consumption is distributed across various systems/sub- systems, components, segments and sub-segments of the network across various services and applications.

A good measurement system will balance accuracy, cost and case of implementation. While it is important to have accuracy, it must be balanced with the cost and case of implementation. For example in a data center, it is possible to measure energy consumption at the data center, rack and system level using direct methods using energy meters and Intelligent power distribution units. It is also possible to estimate power consumption using indirect methods using host utilization 
metrics such as CPU and memory utilization data provided by the operating system. The latter is less accurate but is easy to implement. A good measurement system will also leverage the instrumentation already in place [8].

\section{ROLE OF ICT IN EMISSION REDUCTION}

While climate change issues impose a huge responsibility on the ICT sector to reduce its own carbon footprint intensity and achieve sustainable growth. It also provides unique opportunities for creating an even greater impact by enabling significant emission reduction in other sectors. The Global eSustainability Initiative Report estimates that the ICT sectors can enable emission reduction from BAU levels by almost $15 \%$ - that are about 5 times the ICT sectors own direct carbon footprint.

\section{MAJOR CONTRIBUTIONS OF THE ICT SECTOR IN EMISSION REDUCTION}

\subsection{Smart Power Grids}

The role of ICT in creating Smart Grids cannot be overstated. ICT enables fine grained monitoring of power systems and enables two way communications between end users and power producers While on one hand it enables power producers and distributors to reduce transmission and distribution losses, it also enables consumers to get a better idea of their own consumption and reduce usage. It is possible to shape the demand of consumers via Demand Response. In addition ICT allows integration of renewable sources of energy and distributed generation.

\subsection{Smart Buildings}

Buildings are responsible for roughly $15 \%$ of all emissions. ICT can be used to optimize and monitor the energy consumption right of a building from the design stage to its use. Building automation systems can significantly optimize the use of energy for lighting, heating, ventilation and air-conditioning.

\subsection{Smart Logistics and Intelligent Transportation}

Transportation and Logistics account for nearly 14\% of GHG emissions mainly driven by supply chain Transportation and storage operations. Supply chain execution systems such as Transportation management Systems, route planning and optimization, computer aided dispatch, warehouse management systems, etc., together with technologies such as RFID. Global Positioning System and wireless networks help optimize logistics operations with respect to fossil fuel consumption. Also, emission friendly vehicles such as hybrids and Electric Vehicles will increasingly be used over the years. Intelligent Transportation Systems (ITS \} will help in improving road safety, increase traffic efficiency, improve freight handling and public transit systems and provide driver assistance. All this will lead to less $\mathrm{CO} 2$ emission from vehicles.

\subsection{Smart manufacturing \& Industrial Operations}

$21 \%$ of all GHG emissions are caused by Industrial operations. Big contributors are the chemical and petro-chemicals industries, iron and steel plants, cement plants and manufacturing Process control and automations systems, intelligent motor control \& variable speed drives. It drives energy consumption monitoring, audit \& control. Production planning and ERI are some of the ways in which ICT brings about system efficiencies and hence energy use and emission reduction.

\subsection{Dematerialization}

This refers to substitution of carbon intensive activities with digital alternatives. Examples include teleconferencing and conferencing video conferencing in lieu of face to face meetings, telemarketing and "work-from-home" instead of physical presence in place of work, distance education, telemedicine etc. In addition to this embodied carbon can he reduced via digitization of media such as paper and CD.

\section{THE NINE MAIN PRINCIPLES OF GREEN ENGINEERING}

The nine main principles of green engineering were given as conclusions of the conference "Green Engineering: Defining the Principles" held in Sandestin, Florida, USA, in May 2003. These principles are:

1. Holistic processing and integration of environmental impact assessment tools

2. Conservation and Improvement of natural ecosystems while protecting human health and well-being

3. Using life-cycle thinking in all engineering activities

4. Ensuring that all material and energy inputs and outputs are as inherently safe and benign as possible

5. Minimization of depletion of natural resources

6. Prevention of waste as much as possible

7. Development and application of engineering solutions while being cognizant of local geography, aspirations and cultures

8. Improvement and creation of new and innovative technologies to achieve sustainability;

9. Active engagement of communities and stakeholders in development of engineering solutions.

\section{ROLE OF CLOUD COMPUTING IN CREATING A GREEN ICT INFRASTRUCTURE}

According to the research firm IDC, the following areas were the top five cloud application use in 2010;

- Collaboration application

- Web application/Web serving

- cloud backup

- Business application

- Personal productivity application

As these various datacenters grew in size, businesses have developed their datacenters as "Greenfield" projects. Datacenters have been sited to do the following:

- Have access to low cost power

- Leverage renewable power source

- Be near abundant water

- Be sited where high-speed network backbone connections can be made

- Keep land costs modest and occupation unobtrusive 


\section{- Obtain tax breaks}

- Optimize the overall system latency

Cloud computing focuses towards achieving the objectives stated in the nine main principle of green engineering. These characteristics make cloud computing networks highly efficient and capture enough margin to make utility computing profitable.

A recent research by Accenture shows that Cloud based business applications can lead to reduce carbon footprint of organizations. As per the report, where large corporations can save at least 30 to $60 \%$ in carbon emissions using cloud application, this save will be 60 to $90 \%$ for mid-scale businesses. For small scale business this will be almost $90 \%$ while using cloud based applications [9].

\section{CLOUD COMPUTING IN ORGANIZATIONS \\ 10.1 Microsoft versus Google}

A remarkable battle is emerging between the two IT giants, Microsoft and Google that will have a major impact on the delivery of application software in the future. The Microsoft business is based on the traditional model whereby software is sold to customers for a license fee, ongoing improvements and upgrades commanding additional fees. A dominant market share for PC computing is captured by incorporating the Microsoft operating system and basic application software in the sale of new computers. More than 500 million people around the globe are using Microsoft software through this delivery mode.

Google and other smaller players are now challenging this business model by offering software applications that are delivered through the web. This means that the application computer code resides on the server of the software company, rather than on the user's own computer. These online software products, so-called "software as a service" (SaaS), are not subject to the traditional long build, test and shipping cycles of software development, since the web-based software can be delivered and updated through the web providing an ideal model for "speed to market". For example, Google has updated its e-mail systems, its online applications such as spreadsheets, word processing, presentations and its new cell phone software in just months rather than years. For small businesses, this software online model can also substantially reduce their internal IT support service costs. The growth of this business model is reflected in the fact that some 2,000 small business companies are signing up for Google applications each working day.

Clearly, Cloud computing has the potential to provide even relatively small companies with access to supercomputing power and highly advanced software tools, at relatively low cost through the Internet. Even large corporations are starting to move to Google applications such as Gmail, to provide the services needed to handle large volumes of e-mail traffic and spam.

Mobile phones are destined to have a bright future with about a billion people around the world using this technology as a prime means of interpersonal communication, Following the successful introduction of the first touch technology iPhone in mid-2007, Apple has more recently launched a new and cheaper $3 \mathrm{G}$ network model with a built-in Global Positioning System (GPS) and enhanced computing power and memory.

In addition, Apple released a software developer's kit (SDK) in advance of this launch to enable independent companies to create new applications for the iPhone.

This has led to a surge of software development creating new applications that have turned the iPhone into, for example, a game console, a musical instrument, a navigation tool, a web browser device and a medical imaging viewer.

This new trend towards open innovation around mobile phone software development has major implications for this industry and poses a threat to other players with a closed software innovation business model such as Nokia.

The iPhone may also have far reaching implications for the wider use of the mobile Web for advertising and hence also poses a threat to companies like Google [10].

\subsection{Microsoft's Surface}

Microsoft has launched a technology called Surface, which is a touch sensitive table that could possibly redefine the way people interact with machines. This innovative technology has been running hot and cold in the Microsoft research labs for some five years prior to this market launch.

The Surface technology takes advantage of people's natural sense of touch and spatial orientation, offering more precise control over what's happening on the screen than pointing and clicking with a mouse. Further, the systems can incorporate familiar objects such as toys, game tokens and cell phones into the computing experience. The broader usefulness of Surface computing remains to be seen. Since the current applications appear to be somewhat limited. In addition, the success of tactile computing may depend in large part on how willing the users are to adapt to a new computer interface technology [11].

However, the success of the Apple iPhone, which employs advanced touch technology, may indicate that this form of human machine interaction does indeed have high potential for the future.

\section{CONCLUSION}

Thus cloud computing plays a major role in helping the organizations create a sustainable green information and communication technology infrastructure. Cloud computing has enormous potential to transform the world of IT-reducing costs, improving efficiency and business agility, and contributing to a more sustainable world. Cloud computing can reduce carbon emissions by 30 to 90 percent for major business applications today and that future energy savings are likely as cloud computing continues to evolve. Companies who adopt cloud computing will accrue the inherent business benefits of this technology, and will also play a crucial role in making IT more sustainable by significantly reducing energy consumption.

\section{REFERENCES}

[1] M. Creeger, "CTO Roundtable: Cloud Computing", Communications of the ACM, Vol. 52 No. 8, Pages 50-56 $10.1145 / 1536616.1536633$

[2] L.-J. Zhang and Q. Zhou, "CCOA: Cloud Computing Open Architecture" Proc. IEEE 7th International Conference on Web Services (ICWS'09) pp. 607-616 Jul. 2009

[3] Rakesh Agrawal, Anastasia Ailamaki, Philip A. Bernstein , Eric A. Brewer, Michael J. Carey, Surajit Chaudhuri, Anhai Doan, Daniela Florescu, Michael J. Franklin, Hector Garcia-Molina , Johannes Gehrke , Le Gruenwald , Laura M. Haas, Alon Y. Halevy, Joseph M. Hellerstein , Yannis E. Ioannidis , Hank F. Korth , Donald Kossmann, Samuel Madden , Roger Magoulas, Beng Chin Ooi , Tim 
O'Reilly , Raghu Ramakrishnan , Sunita Sarawagi, Michael Stonebraker, Alexander S. Szalay , Gerhard Weikum, The Claremont report on database research, Communications of the ACM, v.52 n.6, June 2009 [doi> 10.1145/1516046.1516062]

[4] Justin Cappos, Ivan Beschastnikh, Arvind Krishnamurthy , Tom Anderson, Seattle: a platform for educational cloud computing, Proceedings of the 40th ACM technical symposium on Computer science education, March 04-07, 2009 , Chattanooga,

$\mathrm{TN}$, USA [doi>10.1145/1508865.1508905]

[5] E. Michael Maximilien , Ajith Ranabahu , Roy Engehausen, Laura C. Anderson, Toward cloud-agnostic middlewares, Proceedings of the 24th ACM SIGPLAN conference companion on Object oriented programming systems languages and applications, October 25-29, 2009, Orlando, Florida, USA [doi>10.1145/1639950.1639957]
[6] Asimov, I., New guide to Science. London: Penguin, 1987

[7] https://www.computer.org/csdl/mags/co/2010/01/mco2010 010022-abs.html [Accessed 25 Dec, 2016]

[8] Chomsky, N., Knowledge of language: Its nature, origin and use. New York, 1986.

[9] Accenture Microsoft Report. 2010. Cloud computing and Sustainability: The Environmental Benefits of Moving to the

Cloud,

http://gesi.org/files/Reports/AssessmentMethodologyCaste Study_CloudComputingSustainability-Nov2010.pdf. [Accessed 30 Dec, 2016]

[10] Ellul, Jacques, The Technological Bluff. Grand Rapids, MI: Eerdmans, 1990.

[11] Feenberg, A., Technology and the Politics of Knowledge. Bloomington, 1995. 